\title{
TERAPIA DE PRESIÓN NEGATIVA COMO ALTERNATIVA EN EL MANEJO DE LA INFECCIÓN EN CIRUGÍA DE COLUMNA
}

\author{
TERAPIA COM PRESSÃO NEGATIVA COMO ALTERNATIVA NO TRATAMENTO DE INFECÇÃO \\ EM CIRURGIA DE COLUNA
}

\section{NEGATIVE PRESSURE THERAPY AS AN ALTERNATIVE IN THE MANAGEMENT OF INFECTION IN SPINAL SURGERY}

Alberto Aceves Pérez', Giovana Paola Medina Romero², José María Jiménez Avila

\begin{abstract}
RESUMEN
Describir las indicaciones y contraindicaciones de la terapia de cicatrización asistida por presión negativa. Se realiza una revisión de la bibliografía existente en las bases de datos de OVID, PubMed, Cochrane y Medigraphic en relación a la terapia de cicatrización asistida por presión negativa. Es un sistema de cicatrización no invasivo y activo que utiliza presión negativa localizada y controlada, que consta de un apósito especializado que incluye esponja reticulada que lleva a través de un tubo el exudado a un contenedor hermético. Este conjunto forma micro deformaciones en el lecho de la herida, que han demostrado ser causa importante de mayor migración fibroblástica y por consiguiente de tejido de mayor calidad, formación de tejido de granulación y angiogénesis. En relación con la infección, el principal enfoque del tratamiento es drenar todo el material purulento, quitar los desechos, el tejido desvitalizado, los cuerpos extraños y eliminar la etiología de la infección. Las indicaciones de la TPN son: heridas agudas, crónicas, por trauma, quemaduras, úlceras por diabetes o por decúbito y abdomen abierto y se contraindica en heridas con tejido necrótico, osteomielitis no tratada, fístulas no exploradas no entéricas y no se recomienda su colocación en áreas de malignidad. Este método es eficaz para el tratamiento de las infecciones post quirúrgicas profundas de la columna vertebral, utilizándose en promedio de 1 a 4 semanas.
\end{abstract}

Descriptores: Terapia de presión negativa para heridas; Infección; Columna vertebral/cirugía.

\section{RESUMO}

Descrever as indicações e contraindicações do tratamento de ferimentos com pressão negativa. Foi realizada uma revisão da literatura existente nos bancos de dados OVID, PubMed, Cochrane e Medigraphic, referente à cicatrização assistida por pressão negativa. Trata-se de um sistema de cicatrização não-invasivo e ativo, que utiliza pressão negativa controlada e localizada, e consta de compressa especializada que inclui esponja reticulada que conduz o exsudado por uma cânula, até um recipiente hermético. Esse conjunto forma microdeformações no leito da ferida, que comprovadamente são a causa importante de maior migração fibroblástica e, assim, da formação de tecido de melhor qualidade, formação de tecido de granulação e angiogênese. Com relação à infecção, o principal enfoque do tratamento é drenar todo o material purulento, remover os detritos, o tecido desvitalizado, os corpos estranhos e eliminar a etiologia da infecção. As indicações do TPN são: feridas agudas, crônicas, por trauma, queimaduras, úlceras por diabetes ou de decúbito e abdome aberto, sendo contraindicado em feridas com tecido necrótico, osteomielite não tratada, fístulas não-entéricas não exploradas e não se recomenda seu uso em áreas de doença oncológica. Esse método é eficaz no tratamento das infecções pós-cirúrgicas profundas da coluna vertebral, com tempo médio de utilização de 1 a 4 semanas.

Descritores: Tratamento de ferimentos com pressão negativa; Infecção; Coluna vertebral/cirurgia.

\begin{abstract}
To describe the indications and contraindications for negative pressure wound therapy (NPWT). We performed a review of the existing literature on negative pressure wound therapy on the OVID, PubMed, Cochrane and Medigraphic databases. This is a non-invasive and active healing system that uses controlled and localized negative pressure. It consists of a specialized dressing with a crosslinked sponge, and a tube through which the exudate flows into an airtight container. This system forms microdeformations on the bed of the wound, which have been demonstrated to be a major cause of fibroblast migration, resulting in the formation of better-quality tissue, granulation tissue formation, and angiogenesis. In relation to the infection, the main focus of treatment is to drain all the pus, removing debris, devitalized tissue, foreign bodies and the etiology of infection. The indications of NPWT are: acute wounds, chronic trauma, burns, diabetic ulcers, or decubitus and open abdomen. It is contraindicated in wounds with necrotic tissue, untreated osteomyelitis, and unexplored non-enteric fistulas, and its use is not recommended in areas of malignancy. This method is effective for the treatment of post-surgical infections of the vertebral spine, with an average time of use of 1 to 4 weeks.
\end{abstract}

Keywords: Negative-pressure wound therapy; Infection; Vertebral spine/surgery.

1. Cirujano Ortopédico, Servicio de Ortopedia del Centro Médico Nacional de Occidente, IMSS en Guadalajara, Jalisco, México.

2. Médico, Cirujano. Centro Medico Nacional de Occidente, Guadalajara, Jalisco, México.

Trabajo realizado en el Servicio de Ortopedia del Hospital de Especialidades, Unidad Médica de Alta Especialidad, Centro Médico Nacional del Occidente, IMSS en Guadalajara, Jalisco, México. Correspondencia: Josefa Ortiz de Domínguez No. 4193.Col. Benito Juárez. CP 44790. Guadalajara, Jalisco, Mexico. alberthoap@ hotmail.com 


\section{INTRODUCCIÓN}

A pesar del conocimiento de la biología de las heridas y los nuevos tipos de curaciones disponibles, en la actualidad un gran número de heridas aun falla en curar evolucionando a la cronicidad. ${ }^{1-3}$

La terapia de cicatrización por presión negativa (TCPN) es una manera de actuar positivamente en el ambiente de la herida que se encuentra estancada en las fases de inflamación y proliferación, y aún en heridas que se encuentran en un estado agudo para disminuir su complejidad y evitar complicaciones aplicando presión subatmosférica, la terapia reconoce diferentes nomenclaturas incluyendo: curación o terapia subatmosférica (subatmospheric dressing, subatmospheric therapy), técnica de sellado al vacío (vacuum sealing technique), cierre de heridas mediado por vacío (vacuum assisted closure), mechado al vacío (vacuum packing), entre otros..$^{1,4-7}$

Básicamente su mecanismo de acción se basa en tres niveles del proceso de curación de heridas:

1. Remoción del excedente de líquido intersticial;

2. Trasmisión de fuerzas mecánicas que estimulan la proliferación tisular;

3. Mantenimiento de un ambiente adecuado en la herida en cuanto a humedad y temperatura. ${ }^{1,4,8-10}$

Las primeras referencias con respecto a los métodos de curación de heridas se remontan a los "sanadores" egipcios (asu) que al aplicar una curación compuesta por grasa animal, miel y lino, probablemente no sabían que estaban utilizando una curación no adherente, osmótica y antibacteriana y con capacidad de absorción de exudados, respectivamente.

Antes de 1960 el manejo de las heridas estaba dirigido a prevenir la infección por lo cual luego de lavarlas con antisépticos, las mismas se cubrían con una curación seca que evitaría el desarrollo bacteriano.

Se comprobó que las heridas que se mantenían húmedas curaban más rápidamente que las expuestas al aire, desde entonces los beneficios de la curación húmeda han sido probados y son utilizados diariamente en el manejo de heridas.

A medida que la biología de las heridas se ha hecho más conocida, se han definido también las características de la curación ideal. ${ }^{1,7,9,11-13}$

La terapia de cicatrización por presión negativa (TCPN) es un sistema de cicatrización no invasivo, activo que utiliza presión negativa localizada y controlada, esta consta de un apósito especializado que incluye esponja reticulada con una porosidad especifica de 400 a 600 micras la cual es sellada con un apósito semioclusivo cuyo objetivo es mantener la presión negativa en el sitio de la herida, llevando a través de un tubo el exudado a un contenedor hermético con un sobre antibacterial y gelatinizante, esto en conjunto forma en el lecho de la herida micro deformaciones que en diversos estudios han demostrado ser causa importante de una mayor migración fibroblástica y por consiguiente un tejido de mayor calidad, mayor formación de tejido de granulación y angiogénesis. (Figura 1)

La efectividad de este proceso está dado por:

1. Remoción de fluidos:

a. Disminución de factores pro inflamatorios o degradadores de la matriz extracelular;

b. Retiro efectivo de microorganismos contenidos en el lecho de la herida.

2. Aumento del Flujo Sanguíneo Capilar, cuatro veces mayor;

3. Aumento de la Formación de tejido de granulación de hasta 103\%;

4. Promoción de un ambiente húmedo para la cicatrización;

5. Reducción del edema local.

No existe en la actualidad una curación que incluya todas estas características y con el objeto de encontrar esta curación ideal, se han probado varias técnicas y materiales como: alginatos, hidrogeles, colágeno y productos de reemplazo de la piel que han demostrado su utilidad en ciertos tipos de heridas. (Figura 2)

Existen varios dispositivos en etapa de experimentación y aplicación aún no generalizada como: los factores de crecimiento,

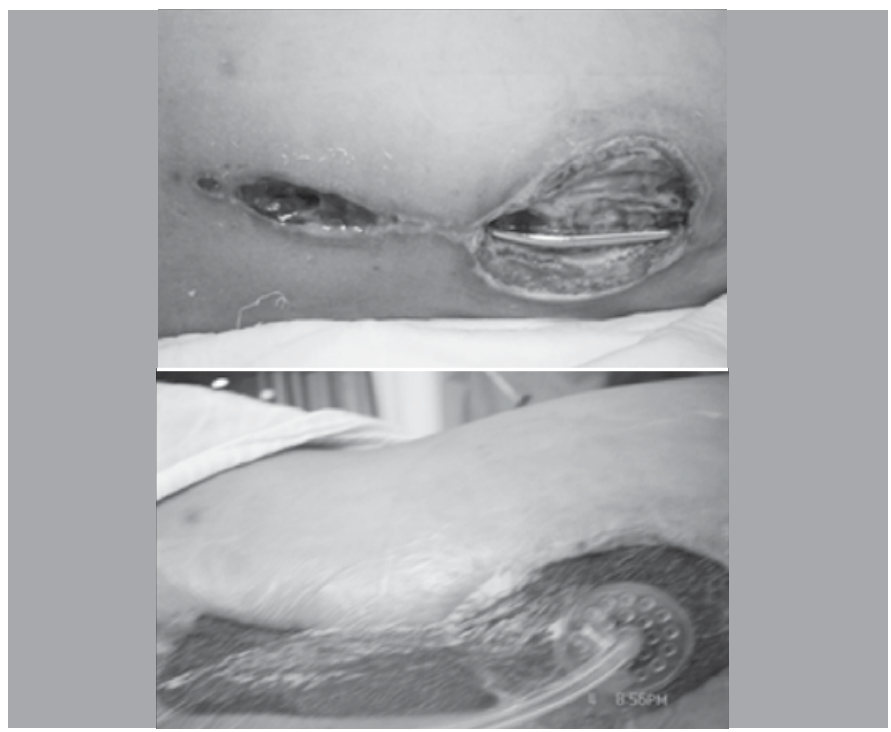

Figura 1. Paciente femenino de con diagnóstico de escoliosis y colocación de barras de Luque cursando con dehiscencia e infección de la herida, tratada con terapia de cierre con presión negativa.

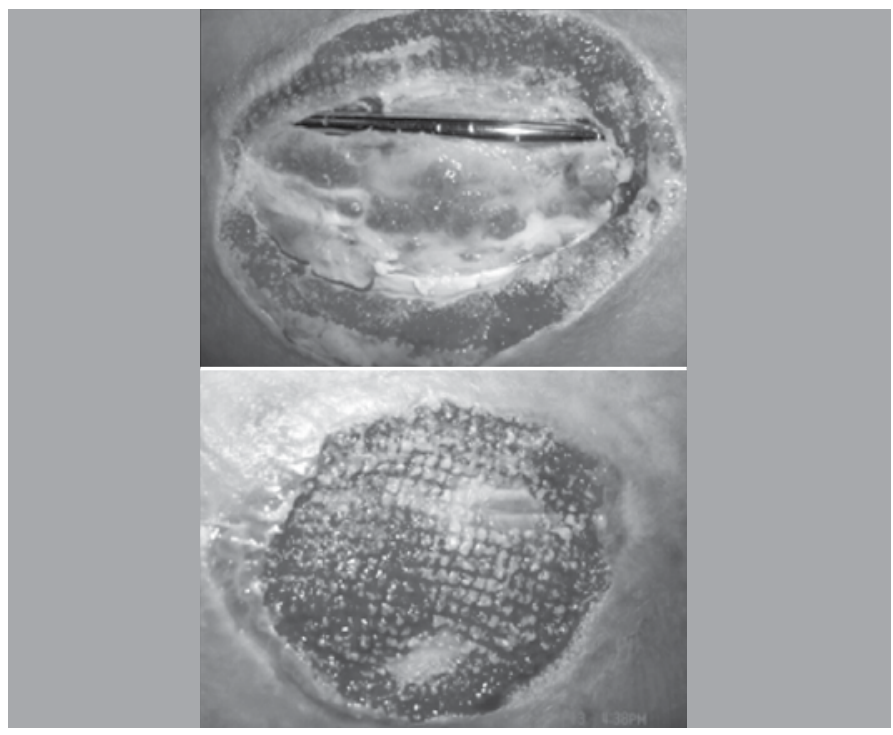

Figura 2. Paciente femenino a las 2 semanas de tratamiento y posteriormente a los 22 días, obsérvese la formación de tejido de granulación, así como la cobertura del material de instrumentación.

agentes farmacológicos, láser, oxigenoterapia hiperbárica y estimulación eléctrica y la mayoría de éstas se consideran como técnicas avanzadas en el cuidado de heridas (advanced wound care technologies). 1,10,12,14

Estas se definen como aquellas que inciden en el proceso de curación de heridas en forma activa y positiva por contrarrestar, eliminar o disminuir significativamente por los menos dos factores que puedan comprometer este proceso.

La terapia de cicatrización de presión negativa (TCPN) utilizada en forma aislada o asociada a otras técnicas similares aumenta las tasas de curación de las heridas. . $^{3,10,15}$

Dado el aumento en el número y expectativa de vida de la población con heridas crónicas, en aquellos que requieren cuidados locales adicionales para llegar a la indicación y oportunidad quirúrgica, o en los que los procedimientos quirúrgicos han fallado, las técnicas avanzadas del cuidado de heridas y entre ellas la TCPN se convierten en la modalidad terapéutica primordial y no raramente la única.,6,7,9

Este tipo de heridas son más probables en el anciano o en las personas con múltiples problemas de salud, debido a que las 
personas están viviendo mucho más tiempo, con cuadros más complejos y están siendo sometidas a más procedimientos de tratamiento quirúrgico extensivos, el número de personas con heridas crónicas y complicaciones de heridas continúa aumentando, a pesar de la atención médica o de enfermería, algunas de estas heridas no curan fácilmente y causan mucha incomodidad y dolor. ${ }^{1,4,16}$

El manejo inicial de las heridas está dirigido a prevenir la infección, por ejemplo, lavando la herida con un antiséptico y cubriendo la herida con un vendaje seco que no contenga bacterias, la investigación sobre cicatrización de heridas en las décadas de 1950 y 1960 reveló que las heridas agudas curan más rápidamente si se mantienen húmedas, ya que esto permite que las células de la dermis y epidermis se dividan y migren por la superficie de la herida., 2, $, 5,12$

A pesar del uso de vendajes modernos, un gran número de heridas crónicas todavía son difíciles de curar, una manera de manipular el medio ambiente de la herida es aplicar una presión negativa tópica (TCPN) (medida en $\mathrm{mm} \mathrm{Hg}$ ) a la superficie de la herida, por medio de un vendaje, el concepto de presión negativa para crear una fuerza de aspiración que posibilite el drenaje de heridas quirúrgicas para estimular la cicatrización de la herida está bien documentado. 1,2,5,8,9,14,17

Se ha sugerido hace mucho tiempo que si el exceso de líquido no es adecuadamente eliminado de la herida después de una operación, sus componentes pueden funcionar como impedimentos físicos y químicos para la cicatrización de la herida.

El concepto básico de que las fuerzas mecánicas influyen sobre la forma y el crecimiento de los tejidos también está bien documentado, se ha informado que la (TCPN) tiene ambas propiedades es decir, quita el exceso de líquido intersticial y transmite fuerzas mecánicas a los tejidos circundantes con la resultante deformación de la matriz extracelular y de las células. ${ }^{8-10,18,19}$

Se supone que ambos factores estimulan la cicatrización de la herida a través de una variedad de mecanismos, el vendaje adhesivo transparente empleado para afianzar la venda también puede ayudar a mantener un ambiente húmedo de la herida.

Existen varios términos para describir el tratamiento de una herida con TCPN, incluyendo tratamiento o vendaje de presión subatmosférica, técnica de sellado al vacío, cierre de herida asistido por vacío, cierre ayudado por vacío, vendaje de presión negativa, vendaje de espuma con aspiración, compresión por vacío, compresa al vacío o aspiración de la herida con superficie sellada, a los fines de esta revisión, la intervención se denominará TCPN. 8,10,12,13,20

La TCPN requiere una venda de celdas abiertas (p.ej., espuma, fieltro, toalla quirúrgica, gasa) para comprimir la herida, una estructura tubular, para conectar el vendaje a una bomba de aspiración con un recipiente intermedio que recoge cualquier exudado que pueda existir y un cierre hermético alrededor del vendaje, sin embargo existe evidencia suficiente que demuestra que los apósitos con porosidad especifica de 400 a 600 micras son los únicos capaces de lograr las micro deformaciones en el tejido.

Todos los tejidos no viables se extirpan de antemano, la TCPN se considera generalmente contraindicada si la herida o los tejidos circundantes son cancerosos, si hay fístulas que comunican con órganos o cavidades, en casos que se sospecha de una fístula de líquido cefalorraquídeo o bien si existe tejido necrótico o una osteomielitis no tratada. 1,3,7

La evidencia de laboratorio acerca del efecto de la TCPN sobre el medio ambiente de la herida se ha obtenido a partir de varios estudios en animales, una serie de estudios, realizados usando un modelo en cerdo de heridas agudas, informó un aumento en la perfusión funcional sanguínea local, una velocidad significativamente superior de formación de tejido de granulación, una reducción significativa de los niveles de bacterias en tejidos y un aumento significativo en el flujo sanguíneo en heridas tratadas con TCPN, comparadas con heridas tratadas con gasa humedecida en solución fisiológica.9,2,12,20,21

El uso de TCPN en heridas humanas crónicas ha sido descrito por una cantidad de clínicos, el uso de TCPN para el tratamiento en el domicilio de heridas crónicas ha sido revisado usando ensayos con grupos control no simultáneo.
Esta revisión examina el impacto de la TCPN sobre las heridas humanas crónicas utilizando ensayos en los que los pacientes se han asignado al azar a grupos control coexistente. 4,12,13,16,19

En relación con la infección, el principal enfoque de tratamiento es drenar todo el material purulento, quitar los desechos, el tejido desvitalizado, los cuerpos extraños y eliminar la etiología de la infección. 4,5

Para el manejo de la herida complicada y de los factores arriba mencionados se recurre a diversas terapéuticas, como la limpieza quirúrgica, el empleo de fármacos de mayor potencia, el oxígeno hiperbárico etc, con resultados aceptables, sin embargo, en muchos casos se requiere un largo periodo de atención médica dentro del hospital, con el consecuente consumo de recursos materiales y humanos: antibióticos, antisépticos, material de curación, horas quirófano, tiempo del médico o personal paramédico, etc. ${ }^{19,22,23}$

La primera función la ejecuta la unidad computarizada que sirve para extraer fluidos de la herida, evacuados hacia un recipiente por medio del tubo colector colocado sobre la esponja, esta acción acelera el proceso de cicatrización, al activar la señal de división celular y por incremento en el flujo sanguíneo, existen dos tipos de esponja la primera es de poliuretano de poro abierto (400 a $600 \mu \mathrm{m}$ ), hidrofóbica, indicada para formación de tejido de granulación siempre y cuando no exista tendones, vasos sanguíneos o nervios expuestos, en estos casos se tendrá que utilizar la esponja hidrofílica de polivinilalcohol, ambas tienen la propiedad de transmitir la fuerza de aspiración a la superficie cruenta, donde provoca micro-deformaciones, se cuenta además con una esponja adicional de las mismas características que la de poliuretano pero con una adición de plata para heridas infectadas. 8,10,11,15

La esponja está diseñada con poro abierto para facilitar la eliminación del exceso de exudado de la herida, transmite la presión subatmosférica a la herida, acción que provoca modificaciones en la superficie del tejido en contacto con ella, induciendo aceleración del proceso de cicatrización, la película adhesiva mantiene ambiente húmedo, con la característica de ser permeable, lo cual facilita el intercambio gaseoso y junto con la esponja, protege la herida de contaminantes del ambiente. 1,12,24,25 (Figura 3)

Al extraer el excedente de líquido de la zona cruenta, disminuye la compresión, lo que favorece al sistema linfático y al circulatorio, la mayor tensión dificulta la oxigenación así como el paso de nutrientes; aislando la herida se elimina o disminuye la carga bacteriana, que consume nutrientes, oxígeno y desnaturaliza proteínas, con daño a la cicatrización. (Figura 4)

Se ha demostrado también que la presión negativa incrementa el flujo sanguíneo por disminución del edema alrededor de la herida y por la remoción de enzimas dañinas del lecho de la herida.,12

Las indicaciones de la terapia TCPN son: heridas agudas, crónicas, por trauma, quemaduras, úlceras por diabetes o por decúbito y abdomen abierto y se contraindica en heridas con tejido necrótico,

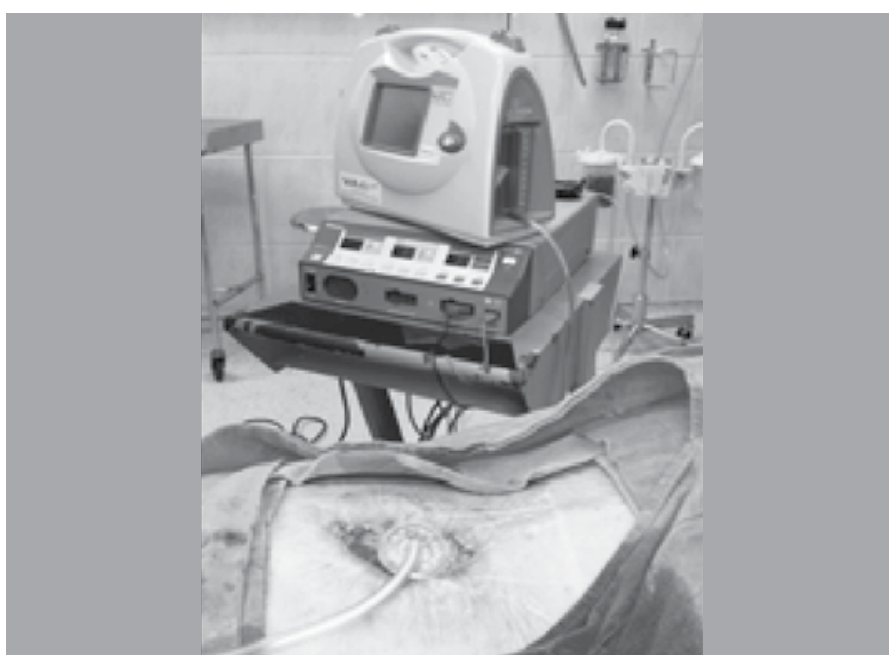

Figura 3. Paciente de 56 años con una discitis posterior a una cirugía de columna. 


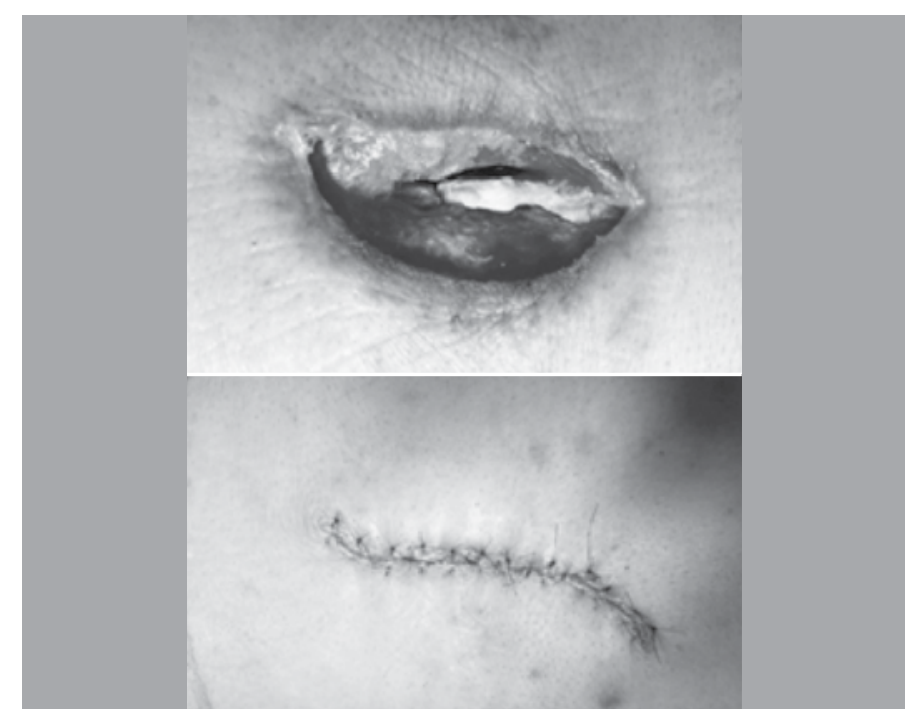

Figura 4. Paciente con dehiscencia de herida quirúrgica en región lumbar, después de 4 recambios y 3 semanas de evolución.

osteomielitis no tratada, fístulas no exploradas no entéricas y no se recomienda su colocación en áreas de malignidad.

Este tipo de terapia ha sido aceptada en la cirugía de columna, su utilidad es de gran ayuda si se considera el elevado costo de la instrumentación utilizada en este tipo de cirugías y una de las contraindicaciones se encuentra la sospecha o presencia de ruptura o desgarro de la duramadre con posible fuga de líquido cefalorraquídeo. ${ }^{1,3,6,7,23}$

\section{CONSIDERACIONES FINALES}

Las infecciones profundas que ocurren posterior a una cirugía de columna son causa muy significativa de morbimortalidad, ya que tienen una incidencia del $0.9 \%$ al $13.4 \%$ de todos los procedimientos quirúrgicos, lo cual representa un problema muy importante tanto para el paciente como para el equipo de salud y los distintos niveles de atención.

La terapia de cierre con presión negativa es usada con gran éxito, principalmente en la cirugía abdominal, cirugía de tórax con apertura esternal y en las fracturas expuestas de miembros inferiores, el cual mediante presión negativa se elimina y absorbe el edema y los líquidos, mejorando la circulación sanguínea y generando la proliferación celular con tejido de granulación, así como factores de crecimiento tisular local.

Además se ha demostrado que el uso de terapia con presión negativa disminuye los costos en la atención del paciente, ya que los ahorros económicos derivados de la cicatrización más rápida, el menor tiempo dedicado por el personal médico y paramédico, la menor estancia hospitalaria y la disminución en el tiempo de uso de antibiótico podrían compensar los costos de adquisición de los apósitos para el tratamiento con presión negativa.

Este método es eficaz para el tratamiento de las infecciones post quirúrgicas profundas de la columna vertebral, utilizándose en promedio de 1 a 4 semanas, influyendo en este tiempo si existe exposición de material inerte, realizando los recambios de la esponja y la película adherente tres veces por semana teniendo un porcentaje significativo de éxito sin retirar el implante expuesto, así mismo realizando repetidos desbridamientos y aseos exhaustivos, hasta el cierre completo de la herida.

La indicación de la TCPN, está dada principalmente por el índice creciente de pacientes que presentan infecciones de heridas quirúrgicas posteriores a una intervención en la columna vertebral. A esto se añade que la terapia ofrece la diminución de los días de estancia hospitalaria, disminución del tiempo de terapia con antimicrobianos, disminución en los costos de la atención médica y restituir al paciente en el menor tiempo posible a sus actividades cotidianas.

La infección asociada a la atención de la salud, conocida como nosocomial, es le infección localizada o sistémica que resulta como una reacción adversa a la presencia de un agente infeccioso.

En Estados Unidos, este tipo de infecciones deroga más de 100,000 muertes al año con un costo de cerca de $\$ 6.7$ billones de dólares al año.

En México, la incidencia de infección en el sitio de la cirugía después de una laminectomía descompresiva, discectomía y fusión es menor a $3 \%$, pero la incidencia se incrementa hasta $12 \%$ cuando el paciente fue instrumentado.

Todos los autores declaran que no hay ningún potencial conflicto de intereses con referencia a este artículo.

\section{REFERENCIAS}

1. Antony S, Terrazas S. A retrospective study: clinical experience using vacuum-assisted closure in the treatment of wounds. J Natl Med Assoc. 2004:96(8):1073-7.

2. Labler $L$, Keel $M$, Trentz $O$, Heinzelmann M. Wound conditioning by vacuum assisted closure (V.A.C.) in postoperative infections after dorsal spine surgery. Eur Spine J. 2006;15(9):1388-96.

3. Morykwas MJ, Faler BJ, Pearce DJ, Argenta LC. Effects of varying levels of subatmospheric pressure on the rate of granulation tissue formation in experimental wounds in swine. Ann Plast Surg. 2001;47(5):547-51.

4. Brox-Jiménez A, Díaz-Gómez D, Parra-Membrives P, Martínez-Baena D, Márquez-Muñoz $\mathrm{M}$, Lorente-Herce J, et al. [A vacuum assisted closure system in complex wounds: a retrospective study]. Cir Esp. 2010;87(5):312-7.

5. Dunn RM, Ignotz R, Mole T, Cockwill J, Smith JM. Assessment of gauze-based negative pressure wound therapy in the split-thickness skin graft clinical pathway-an observational study. Eplasty. 2011;11:e14

6. Nain PS, Uppal SK, Garg R, Bajaj K, Garg S. Role of negative pressure wound therapy in healing of diabetic foot ulcers. J Surg Tech Case Rep. 2011:3(1):17-22.

7. Yuste Be, Rodrigo P, Silva B. Tratamiento mediante presion negativa VAC de herida infectada tras artrodesis raquídea. Cir Plast Iberolatinoam. 2011;37(1):S73-6.

8. Kolios L, Kolios G, Beyersdorff M, Dumont C, Stromps J, Freytag S, et al. Cost analysis of Topica Negative Pressure (TNP) Therapy for traumatic acquired wounds. Ger Med Sci. 2010:8:Doc13.

9. Malmsjö M, Gustafsson L, Lindstedt S, Gesslein B, Ingemansson R. The effects of variable, intermittent, and continuous negative pressure wound therapy, using foam or gauze, on wound contraction, granulation tissue formation, and ingrowth into the wound filler. Eplasty. 2012;12:e5

10. Ortega-León LH, Vargas-Domínguez A, Ramírez-Tapia D, Zaldívar-Ramírez FR, Rodríguez-Báez A, Montalvo-Javé E. Empleo de la presión negativa en el tratamiento de heridas complicadas. Reporte de seis casos. Cir Gen. 2011;33(2):115-20.

11. Hsu CC, Tsai WC, Chen CP, Lu YM, Wang JS. Effects of negative pressures on epithelial tight junctions and migration in wound healing. Am J Physiol Cell Physiol. 2010:299(2):C528-34.

12. Singh $K$, Anderson $E$, Harper JG. Overview and management of sternal wound infection. Semin Plast Surg. 2011;25(1):25-33.

13. Penn-Barwell JG, Fries CA, Street L, Jeffery $S$. Use of topical negative pressure in british servicemen with combat wounds. Eplasty. 2011;11:e35.

14. Armstrong DG, Jude EB. The role of matrix metalloproteinases in wound healing. J Am Podiatr Med Assoc. 2002:92(1):12-8.

15. Canavese F, Gupta S, Krajbich JI, Emara KM. Vacuum-assisted closure for deep infection after spinal instrumentation for scoliosis. J Bone Joint Surg Br. 2008;90(3):377-81.

16. Saxena V, Hwang CW, Huang S, Eichbaum O, Ingber D, Orgill DP. Vacuum-assisted closure: microdeformations of wounds and cell proliferation. Plast Reconstr Surg. 2004;114(5):1086-96

17. Ortiz PN, Remondino R, González G, Catá CE. Infeccion profunda post quirurgica luego de una fusion espinal. Manejo con el sistema de cicatrizacion asistida por vacio. Rev Asoc Argent Ortop Traumatol. 2010;74(4):365-8.

18. Peinemann F, Sauerland S. Negative-pressure wound therapy: systematic review of randomized controlled trials. Dtsch Arztebl Int. 2011;108(22):381-9.

19. Macedo FB, Carriquiry CE. Tratamiento de heridas utilizando presion negativa tópica. Biomedicina. 2006:2(2):122-30.

20. Gwan-Nulla DN, Casal RS. Toxic shock syndrome associated with the use of the vacuumassisted closure device. Ann Plast Surg. 2001;47(5):552-4.

21. Moffatt CJ, Soldevilla JJ. La presion negativa topica en el tratamiento de heridas. Documento de Posicionamiento. GNEAUPP. 2007;7:1

22. Venturi ML, Attinger CE, Mesbahi AN, Hess CL, Graw KS. Mechanisms and clinical applications of the vacuum-assisted closure (VAC) Device: a review. Am J Clin Dermatol. 2005;6(3):185-94.

23. Jimenez-Avila JM, Calderon-Granados A, Bitar-Alatorre W. Costo directo de las lesiones en columna. Cir Cir. 2012:80:435-41.

24. Shi B, Chen SZ, Zhang P, Li JQ. [Effects of vacuum-assisted closure (VAC) on the expressions of MMP-1, 2, 13 in human granulation wound]. Zhonghua Zheng Xing Wai Ke Za Zhi. 2003;19(4):279-81.

25. Horn PL, Ruth B, Kean JR. Use of wound V.A.C. therapy in pediatric patients with infected spinal wounds: a retrospective review. Orthop Nurs. 2007;26(5):317-22. 